\title{
Message Scheduling Algorithm Design for Switched FlexRay Network
}

\author{
Yi-Nan Xu, Xiang-Qi Kong, Meng-Zhuo Liu, Shu-Wen Xue, and Zhe-Yu Sun
}

\begin{abstract}
As the improvement of vehicle automation technology, it respects high standards in the field of in-vehicle communication. Because of the fast transmission speed and reliability of the FlexRay network, it has become the most popular in-vehicle communication protocol. The topologies of FlexRay is various, including linear, star and hybrid topology structure, it also supports two-channel transmission. On the base of these advantages, the switched FlexRay network is put forward which can extend the system bandwidth and reliability. This paper designs the scheduling algorithm for the switched FlexRay network, and one new method which can precisely compute the value of the worst case response time of DYN messages is presented. The experiments with computer simulations prove the efficiency and improvements of the proposed scheduling algorithm, and the results show that the worst case response time computing method is better than previous methods.
\end{abstract}

Index Terms-FlexRay, scheduling, static segment, dynamic segment, worst case response time.

\section{INTRODUCTION}

The modern vehicle electronic system consists of Electronic Control Units (ECUs) which are embedded in the fundamental components of the vehicle, such as, microprocessors, sensors and actuators. The information communications between the ECUs constitute the vehicle communication network [1]. Nowadays, the ECUs' number of the upscale vehicles exceeds 70 and signals' quantity reaches 2500 . The first predominant communication system in automotive network is Controller Area Network (CAN) [2] It can provide $500 \mathrm{Kbps}$ bandwidth and is based on event-triggered architecture. Because of the natures of CAN networks, it has many limitations in many applications such as electronic components of the power train or $\mathrm{x}$-by-wire applications. In 2000, the FlexRay network as one industry consortium was proposed by BMW, Kreisler, Philips and Freescale, and the number of the consortium is over 150 [3-4] The fast speed and stability features make it become the most popular in-vehicle communication framework in modern industry fields. FlexRay has one static segment (SS) and one dynamic segment (DYN). As the time-triggered feature of FlexRay, it becomes the bus standard for X-by-wire systems that requires fast speed and high safety.

The scheduling scheme of the in-vehicle communication

Manuscript received March 3, 2017; revised April 13, 2017. This work was supported by the National Natural Science Foundation of China (61361003)

The authors are with the College of Engineering of Yanbian University, Yanji, 133002, China (e-mail: ynxu@ybu.edu.cn, ). system as one hot topic attracts many researchers' attentions. In the early days, the works of focus on testing the feasibility of the given schedule for FlexRay network [5-7]. The further works present more efficient feasible schedule [8]-[10]. The switched FlexRay network as one new communication structure which can extend the bandwidth of the system is presented [11]. The switch module allows several message set can be transmitted simultaneously [12], [13]. In recent years, the research about the scheduling of the switched FlexRay network has got plenty of good results. Many efficient methods are presented, such as, best-slot-first heuristic, the genetic algorithm and other effective algorithms [14]-[17]. On the base of the present researches, this paper put forward the improved switched FlexRay network scheduling algorithm and one new method which can calculate the worst case response time.

This paper is organized as follows. Section 2 describes FlexRay Protocol and Topologies. Section 3 presents switched FlexRay ST and DYN segment scheduling algorithm design, and the worst case response time compute method in detail. Section 4 contains experimental results and Section 5 the concluding remarks.

\section{PROBLEM DEFINITION}

The high transmission rate and good reliability performance of FlexRay network benefit from its special communication protocol and topologies. Compared with the other in-vehicle communication protocols, such as, CAN and TTP, FlexRay has obvious improvements in many aspects. FlexRay network also supports two-channel transmission, this nature extend the system bandwidth and improve the fault tolerance. Its maximum data rate in the two-channel can reach $10 \mathrm{Mbps}$, the bus data rate reaches $20 \mathrm{Mbps}$. The FlexRay cycle structure is shown as Figure 1. The time measurement of FlexRay communication protocol is Communication Cycle (CC). It offers a maximum of 64 CCs. Each CC includes Static (ST) segment, Dynamic (DYN) segment, Symbol window and network idle time. The static segment is used to transmit important real-time data, ECUs can transmit frame data in bus. There are three parts in the frame, header segment, payload segment and trailer segment.

The communication approach of FlexRay is time-division multiple access (TDMA). During the transmission, the nodes start from left to right. There is at least one unique time interval, called slots. All slots are of same size in the static segment. For the DYN segment, the communication scheme is Flexible TDMA (FTDMA), and it is divided into small time interval, called mini-slots. The symbol window is used to transmit special symbol, such as the wakeup and startup 
symbol of transmit node. The Network Idle Time (NIT) is the unoccupied area, the length of NIT is the maximum macroticks which are not occupied by the other three parts in one CC.

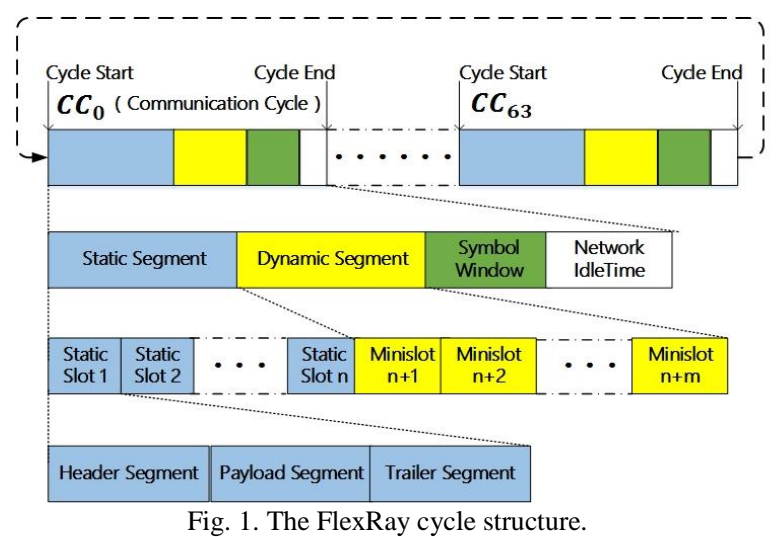

The topology structures of FlexRay are various and convenient to configure. There are three popular topology structures. They are passive linear topology, star topology and hybrid topology. The star topology is divided into passive star, active star and cascade active star. Passive star is one special case of passive linear topology; its bus length is 0 . In the active star topology, each node connects with the central star as one branch, it is same as the communication principle of repeater in the internet, and the active star receives one branch data and broadcasts it. There are at least two branches in one star. When active star number of the network is 2 or more, it becomes the hybrid active network system. The hybrid network is widely used in many vehicles, such as the Audi in-vehicle system, each branch allows the connections of 3 nodes [10].

\section{SWITCHED FLEXRAY NETWORK}

The main difference between simple FlexRay network and switched FlexRay network is the switch module, by replacing the active star with the switch module, the switched FlexRay network can transmit messages in multi-path simultaneously.

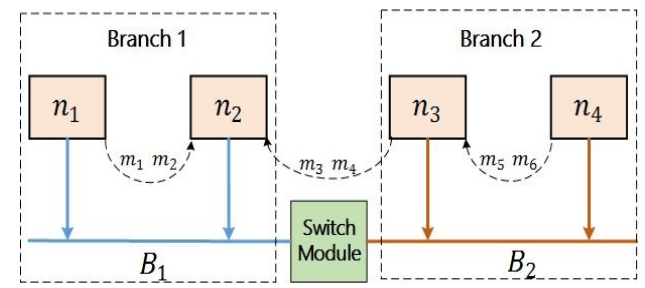

Fig. 2. he FlexRay network with four nodes $n=\left\{n_{1}, n_{2}, n_{3}, n_{4}\right\}$ and a switch module.

\begin{tabular}{|c|c|c|c|}
\hline \multicolumn{4}{|c|}{ (a) Scheduling without FlexRay switch } \\
\hline & Slot 1 & Slot 2 & Slot 3 \\
\hline$B_{1} \cup B_{2}$ & $m_{1} m_{2}$ & $m_{3} m_{4}$ & $m_{5} m_{6}$ \\
\hline \multicolumn{4}{|c|}{ (b) Scheduling with FlexRay switch } \\
\hline & Slot 1 & Slot 2 & \multirow{3}{*}{$\cdots$} \\
\hline$B_{1}$ & $m_{1} m_{2}$ & \multirow{2}{*}{$m_{3} m_{4}$} & \\
\hline$B_{2}$ & $m_{5} m_{6}$ & & \\
\hline
\end{tabular}

Fig. 3. The scheduling results of the network in Fig. 2. Three used slots in (a) and two used slots in (b).
In order to illustrate the advantages of the switched FlexRay network, we present one simple whose structure is shown as Figure 2. There are 4 nodes in the network and they are separated into two branches $B_{1}=\left\{n_{1}, n_{2}\right\}$ and $B_{1}=\left\{n_{3}, n_{4}\right\}$.The message set in the network is $M=\left\{m_{1}, m_{2}, m_{3}, m_{4}, m_{5}, m_{6}\right\}$. Assuming that messages $m_{1}, m_{2}$ and $m_{5}, m_{6}$ can be transmitted in a single slot, the used slots number of simple FlexRay scheduling is 3 . In the switched FlexRay network, messages $m_{1}, m_{2}$ and $m_{5}, m_{6}$ messages can be transmitted at the same slot, the used slots number is 2. The number of used slots in switched FlexRay scheduling is less than the used slots number in simple FlexRay network. Hence, the switched FlexRay network extends the system bandwidth.

\section{SWITCHED FLEXRAY NETWORK}

The messages transmitted in the switched FlexRay ST segment are called switched ST message. According to the difference of message transmission, the switched ST message can be divided into two types. One is the local communication messages which don' $\mathrm{t}$ communicate with the switch; the other is called switched messages which are transmitted through the switch. The source and receiver nodes of switched messages are in different branches. On the contrary, the local communication messages' source and receiver nodes are in the same branch. In the paper, the symbol $M_{m}$ represents the switching paths of message $m$ Then, the switched ST message can be represented by one 4-tuple vector.

$$
m=\left(T_{m}, L S T_{m}, P_{m}, M_{m}\right)
$$

where, $P_{m}$ is the set of source and receiver ports of message $m, T_{m}$ is the minimum inter-arrival time and $L S T_{m}$ is the length of message $m$. And local communication message is one special case of switched message, when, $M_{m}=0$ the switched messages become the local communication message. The other important notations used in switch FlexRay network are presented as follows:

(1) $b_{m}$ : Base cycle is the value of the cycle counter in which the first message arrival sends with the constraints $b_{m} \in[0,63], b_{m} \in Z$.

(2) $D_{m}:$ Message $m$ 's deadline is the duration from message generation to message expiration.

(3) $P_{m}:$ Slot repetition is the multiple of ST slots in between two successive message arrivals within one $\mathrm{CC}$ with the constraints $P_{m} \in[1,1022], P_{m} \in Z$.

(4) $r_{m}$ : Cycle repetition is the multiple of CC in between two successive transmission cycles in which message arrivals send. It has the constraint $r_{m}=2^{n}, n \in[0,6], n \in Z$ to allow a periodic occurrence in the 64 cycles.

(5) $s_{m}$ : Base slot is the value of the ST slot counter in which the first message arrival sends with the constraint $s_{m} \in[1,1023], s_{m} \in Z$. 
Switching matrix table is the set of switching matrix in different slots and in different CCs. Hence, the number of matrix in the table depends on the number of ST slots and CCs. Each matrix represents the connection information of the switching ports. One switching matrix example for communication cycle number $\mathrm{CN}$ and slot ID SID is shown as following.

$$
M_{C N, S I D}=\left(\begin{array}{cccc}
0 & P_{1,2} & P_{1,3} & P_{1,4} \\
P_{2,1} & 0 & P_{2,3} & P_{2,4} \\
P_{3,1} & P_{3,2} & 0 & P_{3,4} \\
P_{4,1} & P_{4,2} & P_{4,3} & 0
\end{array}\right)
$$

Matrix $M_{C N, S I D}$ is the switching matrix of the switch with four ports. Where, the column of the matrix represents the source port, and the row represents the receiver port. Each receiver port may have local communication message or switched communication message.

There are two main constraints in switched FlexRay network. The first one is the schedulable constraint, the other one is the clustering constraint. The schedulable constraints are shown as follows.

(1) The message transmission has to finish before the message deadline $D_{m}$. The worst case response time is smaller than deadline $D_{m}$.Where, $M_{S T}$ represents the ST message set in a cluster. $R_{m}$ indicates the worst case response time of message $m$. This constraint is the most basic requirement for all the schedulable system. The mathematic expression is:

$$
\forall m \in M_{S T}, R_{m} \leq D_{m}
$$

(2) The message transmission has to finish before the next message arrival time. In order to avoid buffer overwriting, the message deadline $D_{m}$ has to be smaller than inter-arrival time $T_{m}$. The mathematic expression is:

$$
\forall m \in M_{S T}, D_{m} \leq T_{m}
$$

The goal of the switched ST scheduling is to increase the system capacity by trying to minimum the number of transmission slots. The direct method is that trying to merge different slots' transmission into one slot as much as possible. The clustering constraints are listed in the following.

(1) A port cannot be the source and receiver port at the same time. The constraint is listed in the following Equation.

$$
\sum_{i=1}^{n} P_{i, i}=0
$$

(2) A receiver port cannot have more than one source port. The constraint is listed in the following Equation.

$$
\forall x \in n: \sum_{i=1}^{n} P_{i, x}>0 \Rightarrow \sum_{j=1}^{n} P_{x, j}=0
$$

(3)A branch that transmits local communication messages during one slot needs to disconnect with the switch. The constraint is listed in the following Equation.

$$
\forall x \in n: \sum_{i=1}^{n} P_{i, x}=1 \vee 0
$$

There are two essential requirements in switch FlexRay network scheduling algorithm. The scheduling algorithm has to satisfy the clustering constraints and schedulable constraints at the same time. The scheduling algorithm structure has two steps. The first step is clustering and generating the switch matrix $M_{C N, S I D}$ of each ST slot, it allocates the ST messages efficiently. The next step is to find the optional scheduling scheme to minimum the system ST slot utilization rate. The main principle of switch FlexRay network is to allocate the most inflexible message. In order to describe the flexibility, one measurement parameter is defined. We use the size of searching space to represent the flexibility, $V_{m}=\left(S_{m}, C C_{m}\right)$,where, $S_{m}=\left[0, p_{m-1}\right]$ and $C C_{m}=\left[0, r_{m-1}\right]$. Hence, the size of flexibility is $p_{m} \times r_{m}$. The conclusion can be got that the scheduling order follows the ascending order of the values of $p_{m}$ and then the ascending order of values of $r_{m}$.

\section{FlexRAy DYN SEgMENT SChEDUling AlgORITHM DESIGN}

The same as switched ST messages, the switched DYN messages are the messages transmitted in the switched FlexRay network. The DYN segment is different with ST segment in two aspects. Firstly, the slot length of ST segment is fixed, the DYN segment slot length is changed depending on the size of the message. Secondly, the message response time is different. The response time of the switched DYN message could be affected by the other messages during the transmission. On the contrary, the switched ST message response time can prevent the interferences by using the fixed slot. In order to describe the switched DYN messages, we define a 4-tuple vector to represent it.

$$
m=\left(D_{m}, L D Y N_{m}, P_{m}, M_{m}\right)
$$

where, $D_{m}$ is the message deadline, $p_{m}$ is the set of the source and receiver ports. And $M_{m}$ and $L D Y N_{m}$ represent the message switching paths and the length of DYN segment message, respectively. The switched FlexRay DYN schedulable constraints and clustering constraints are similar to the constraints of switched FlexRay ST. In DYN segment, the packing period is multiple of the mini-slot. And two different methods are presented to choose the packing period. The first one is packing the cluster every CC. It means that using the duration of the $\mathrm{CC}$ as the packing period. The other one is the flexible packing period, the goal of the second method is to refine the clusters by reducing the set of the branches belong to one cluster.

The other important problem in switched DYN segment scheduling is calculating the worst case response time. The worst case response time is the longest delay time from be produced to be sent. Such as message $\mathrm{m}$ is produced in cycle 
1 , missed its send slot and delayed to be sent in cycle $f$ because of higher-priority message $m$ in figure 4. So the worst case response time consists of three parts, the longest delay time of each part is considered. The following equation is the worst case response time(WCRT) of message $m$.

$$
\begin{gathered}
W C R T(m)=\alpha+(f-1) T_{c}+\operatorname{delay}^{f}(m) \\
J_{m}=\operatorname{ceil}\left(D_{m} / T_{c}\right)
\end{gathered}
$$

Where, $D_{m}$ is the deadline of message $m, T_{m}$ is the FlexRay communication cycle time. $\alpha$ is the longest delay time in its produced cycle. $J_{m}$ is last cycle that message $m$ can be sent. if, $f \leq J_{m}$ then message $m$ can be transmitted before deadline; otherwise not.

$$
\alpha=\left(N_{M S}-\sigma_{I D}(m)+1\right) t_{M S}+T_{S W}+T_{N I T}
$$

where, $N_{M S}$ is minislot number in DYN segment, $\alpha_{I D}(m)$ is the send slot of message $m \cdot t_{M S}$ is the time of one minislot, $T_{S W}$ is the symbol window time, $T_{N I T}$ is the network idle time.

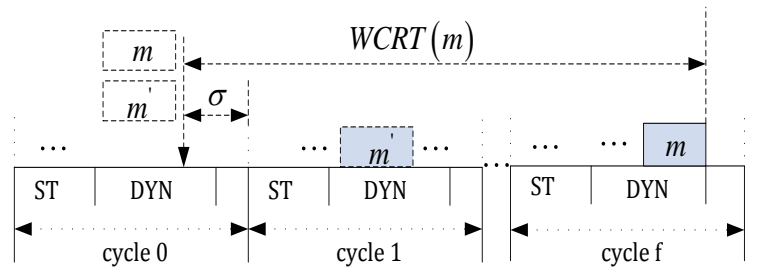

Fig. 4. Worst case response time of dynamic message.

delay $f(m)$ is the delay time in cycle $f$. It contains the time of ST segment, the time before slot $\alpha_{I D}(m)$ and the transmit time of message $m$.

$$
\operatorname{delay}^{f}(m)=T_{S S}+J^{f}(m)+L_{D S}(m) \times t_{M S}
$$

where, $T_{S S}$ is the time of static segment, $L_{D S}(m)$ is the minislot number when sends message in the dynamic segment. Because of the time of $T_{S S}$ and $L_{D S}(m) \times t_{M S}$ are constant, so we just have to know the maximum. $J f(m)$.

To accurately calculate the transmission of $m$ ' which has higher priority than message $m$. A binary variable $x_{m^{\prime} i},\left(1 \leq i \leq J_{m}\right)$ is proposed, and $\alpha_{I D}\left(m^{\prime}\right)<\alpha_{I D}(m)$.

$$
x_{m^{\prime}, i}=\left\{\begin{array}{c}
1, \text { message } m^{\prime} \text { is sent in cycle } i \\
0, \text { message } m^{\prime} \text { is not sent in cycle } i
\end{array}\right.
$$

The times that message $m^{\prime}$ is produced to be sent in $\mathrm{j}$ cycles is $\operatorname{ceil}\left(j \times T_{c} / P_{m^{\prime}}\right),\left(1 \leq i \leq J_{m}\right)$, and $P_{m^{\prime}}$ is the minimum duration between continuous occurs of message $m^{\prime}$. So the transmission of message $m^{\prime}$ in each cycle can be abstracted to an linear integer program.

$$
\left\{\begin{array}{c}
x_{m^{\prime}, 1}+\cdots+x_{m^{\prime}, j} \leq \operatorname{ceil}\left(j \times T_{c} / p_{m^{\prime}}\right) \\
x_{m^{\prime}, 2}+\cdots+x_{m^{\prime}, j+1} \leq \operatorname{ceil}\left(j \times T_{c} / p_{m^{\prime}}\right) \\
\vdots \\
x_{m^{\prime}, J_{m-j+1}}+\cdots+x_{m^{\prime}, J_{m}} \leq \operatorname{ceil}\left(j \times T_{c} / p_{m^{\prime}}\right)
\end{array}\right.
$$

Because of message $\mathrm{m}$ is sent in cycle $J_{m}$ at the earliest, so the time before cycle $J_{m}$ does not satisfy transmit condition that is message $m$ cannot be sent in cycle $\left(J_{m}-1\right)$ and before. The constraint condition is :

$\left.(p \operatorname{Lastest} T x(m)-1)<t_{M S}\left(1-x_{m^{\prime}, i}\right)+L_{D S}\left(m^{\prime}\right) t_{M S}\right\rfloor x_{m^{\prime}, i} \leq T_{D S}$

where, $1 \leq i \leq\left(J_{m}-1\right), L_{D S}\left(m^{\prime}\right) t_{M S} \int x_{m^{\prime}, i}$ is the transmission of message $m^{\prime}$ in cycle I; $t_{M S}\left(1-x_{m^{\prime}, i}\right)$ express message $m^{\prime}$ dose not be sent in its corresponding slot; pLastest Tx $(m)$ is the latest slot that can be sent in each cycle; $T_{D S}$ is the time of dynamic segment .

Because the time before cycle $J_{m}$ is constant, so the worst case response time is decided by the transmission in cycle $J_{m}$, and the longest delay time in cycle $J_{m}$ is as follows:

$$
\left.J f(m)=\max \left(t_{M S}\left(1-x_{m^{\prime}, f}\right)+L_{D S}\left(m^{\prime}\right) t_{M S}\right\rfloor x_{m^{\prime}, f}\right), f=J_{m}
$$

With the up formulas, the worst case response time of message $m$ can be got.

\section{EXPERIMENTAL RESULTS}

To evaluate our message scheduling method, we applied it to Electronic Damper Control (EDC) system which has 4 nodes in BMW X5.The experiments use the following FlexRay configuration settings in table 1.Without loss of generality, we randomly generate 100 sets of ST messages, and each set that represents a different system has different number ST messages, the length of each ST message is equal to 64 bits. The 100 sets of messages are both inputted to our switched message scheduling method and normal message scheduling method, the result is shown in Fig. 5 and 6.

\begin{tabular}{cccccc}
\multicolumn{5}{c}{ TABLE I: FleXRAY NETWORK PART PARAMETER VALUES } \\
\hline Name & Value & Unit & Name & Value & Unit \\
\hline $\mathrm{T}_{\mathrm{SW}}$ & 1 & MT & $\mathrm{T}_{\mathrm{NIT}}$ & 11 & MT \\
$\mathrm{T}_{\mathrm{SS}}$ & 3564 & MT & $\mathrm{T}_{\mathrm{DS}}$ & 60 & $\mathrm{MT}$ \\
$\mathrm{t}_{\mathrm{bit}}$ & 0.1 & us & $\mathrm{t}_{\mathrm{MT}}$ & 1.735 & us \\
$\mathrm{t}_{\mathrm{MS}}$ & 5 & MT & $\mathrm{T}_{\mathrm{C}}$ & 5 & $\mathrm{~ms}$ \\
\hline
\end{tabular}

The used ST slots number is the maximum ST slot ID used by a set of ST message transmission. By summing up the used ST slots in the systems and averaging them by the number of systems, the average used ST slots number can be got. As in Fig. 5 the average used ST slots number of our switched message scheduling methods much less than that of the normal message scheduling method, this is because two 
ST messages that have different ports can share one slot to be sent.

The average network utilization is that can show the system resource used condition. The network utilization is calculated by formula $5-1$.

$$
u=n_{\text {slot }} \times l_{\max } / T_{c}
$$

where, $n_{\text {slot }}$ is the used ST slot number, $l_{\max }$ is the maximum message length.

By averaging systems' network utilization , the result can be seen in figure 6 , where NM is represent for the normal message scheduling method and SM is represent for the switched message scheduling method. It shows that the average network utilization in the switched message scheduling method is less than that in the normal message scheduling method, so this indicates that the switched message scheduling method has better ability to save the system resource.

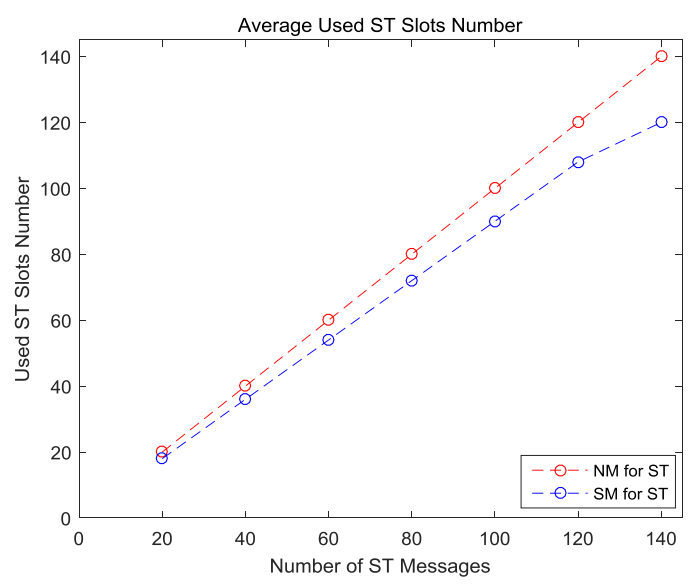

Fig. 5. Average used ST slots number.

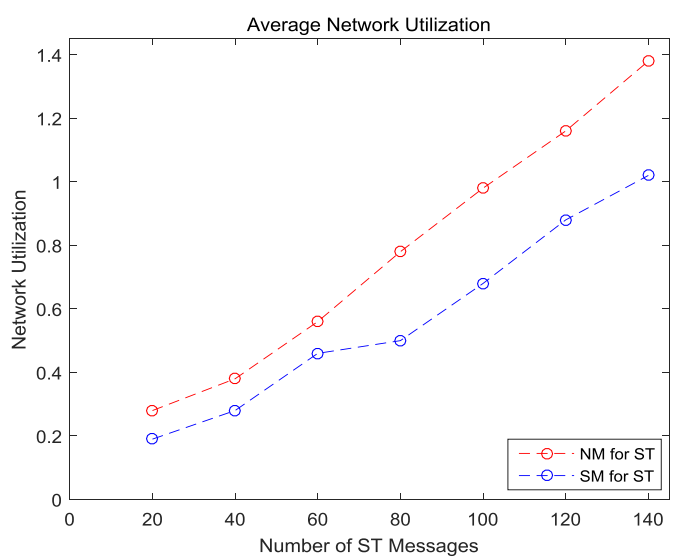

Fig. 6. Average network utilization.

For the DYN segment, the key characteristic is the worst case response time of DYN messages. We present a new method which can compute more precisely than that in paper [18]. The method in paper does not consider DYN message' continuous arrival time and may calculate the worst case response time roughly lead to schedulable messages as unschedulable[18]. 100 sets of DYN messages are put into systems the result can be seen in figure 7 which shows the percentage of scheduled messages is better than paper at the same condition[18] .
The worst case response time of both methods can be seen from figure8. $14 \mathrm{DYN}$ messages with different length are used to evaluate the proposed switched message scheduling method. It is clear to tell from figure 8 that the worst case response times of the switched message scheduling method are equal or less than the normal method, so the worst response time of the DYN messages that can share slots and have large frame IDs can be decreased by our method.

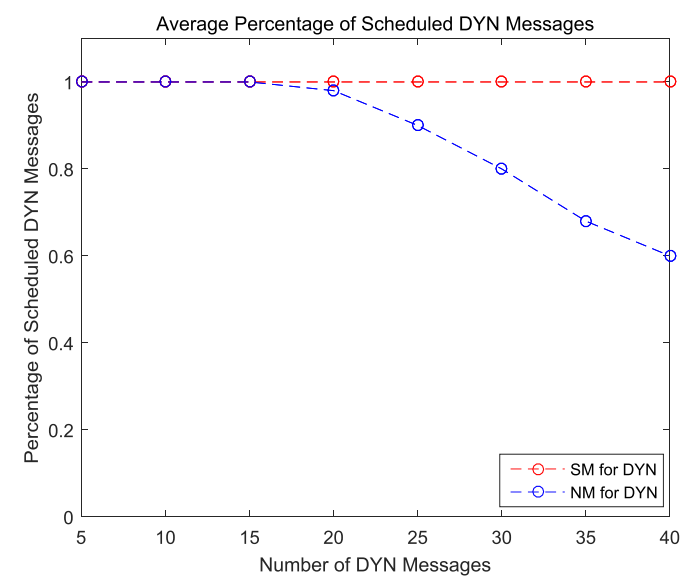

Fig. 7. Percentage of scheduled DYN messages.

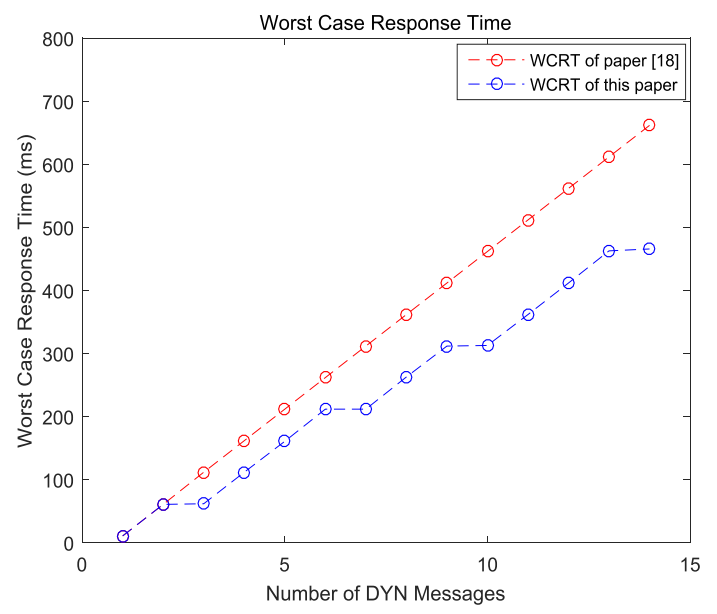

Fig. 8. Worst case response time of DYN message.

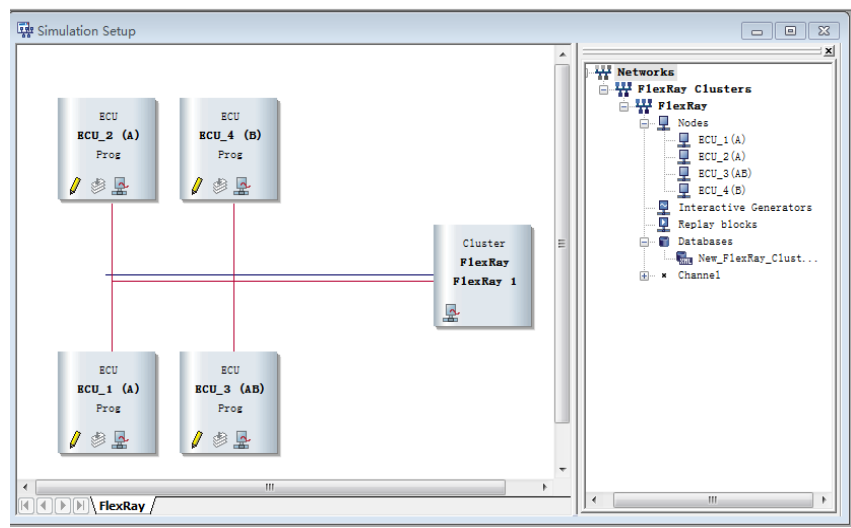

Fig. 9. The node set of FlexRay simulation system.

The Simulation system is evaluated in CANoe and DaVinci Network Designer. ECU-1, ECU-2, ECU-3 and ECU-4 are established in CANoe as show in Fig9. ECU-1 and ECU-4 are transmission nodes and ECU-2 is a receiving node. ECU-3 can be both a transmission node and a receiving node. FlexRay network part parameters are shown in Table1. We use one channel to simulated the simple FlexRay network, 
the message scheduling is shown in Table II. Two channel are used for switch FlexRay network, the message scheduling is

shown in Table III.

TABLE II: THE MESSAGE SCHEdULING OF SimPLE FLEXRAY NeTWORK

\begin{tabular}{l|c|c|c|c|c}
\hline \multirow{2}{*}{ Name } & Transmit & \multicolumn{4}{|c}{ Channel A } \\
\cline { 2 - 5 } & Receive & ST message & Slot & DYN message & Slot \\
\hline \multirow{2}{*}{ ECU-1 } & 1.2 .3 .4$. & 1.2 .3 .4$. & 11.12. & 11.12. \\
\cline { 2 - 5 } & & & & \\
\hline \multirow{2}{*}{ ECU-2 } & & & & \\
\cline { 2 - 5 } & 1.2 .3 .4 .5 .6$. & 5.6. & 15.16. & 15.16. \\
\hline \multirow{2}{*}{ ECU-3 } & 5.6. & & 17.18. & \\
\hline \multirow{2}{*}{ ECU-4 } & 7.8 .9 .10 & 7.8 .9 .10 & 17.18. & 17.18. \\
\cline { 2 - 5 } & & & & \\
\hline
\end{tabular}

TABLE III: THE MESSAGE SCHEDULING OF SWITCH FLEXRAY Network

\begin{tabular}{|c|c|c|c|c|c|c|c|c|c|}
\hline \multirow{2}{*}{ Name } & Transmit & \multicolumn{4}{|c|}{ Channel A } & \multicolumn{4}{|c|}{ Channel B } \\
\hline & Receive & ST message & Slot & DYN message & Slot & ST message & Slot & DYN message & Slot \\
\hline \multirow{2}{*}{\multicolumn{2}{|c|}{ ECU-1 }} & 1.2.3. 4. & 1.2.3. 4 & 11.12. & 7.8. & & & & \\
\hline & & & & & & & & & \\
\hline \multirow{2}{*}{\multicolumn{2}{|c|}{ ECU-2 }} & & & & & & & & \\
\hline & & 1.2.3.4.5.6. & & & & & & & \\
\hline \multirow{2}{*}{\multicolumn{2}{|c|}{ ECU-3 }} & 5.6. & 5.6. & 15.16. & 9.10 & & & & \\
\hline & & & & & & 7.8 .9 .10 & & 17.18. & \\
\hline \multirow{2}{*}{\multicolumn{2}{|c|}{ ECU-4 }} & & & & & 7. 8.9.10 & 1.2.3. 4 & 17. 18. & 7. 8. \\
\hline & & & & & & & & & \\
\hline
\end{tabular}

When the database is built and imported into the CANoe, we can run the Simulation system. The paper simulates simple algorithm and switch algorithm. The network performance comparison are shown in Table4. The switch network decreases the slot number and the payload. The transmission speed increases very quickly.

TABLE IV: NETWORK PERFORMANCE COMPARISON

\begin{tabular}{lll}
\hline Network performance & Simple network & Switch network \\
\hline Slot number & 18 & 10 \\
Frames [f/s] & 2300 & 2900 \\
Busload [\%] & 96.62 & 74.51 \\
\hline
\end{tabular}

\section{CONCLUSION}

This paper carries out the message scheduling algorithm design for switched FlexRay network. The switched message scheduling methods for ST and DYN segment, and the FlexRay network is optimized. Then the test data generated from actual data is used to compare two types of messages scheduling methods for the ST and DYN segment respectively, we improve the bandwidth and reduce the worst case response time of the DYN messages. The simulation results show that transmission speed improves $26 \%$, the slot number decreases by $44 \%$ and the busload decreases by $23 \%$.

\section{REFERENCES}

[1] A. Albert and R. B. Gmbh, "Comparison of event-triggered and time-triggered concepts with regard to distributed control systems," in Proc. Embedded World, 2004, pp. 235-252.

[2] N. Navet, Y. Song, F. Simonot-Lion et al., "Trends in automotive communication systems," in Proc. the IEEE, vol. 93, no. 6, pp. 1204-1223, 2005.

[3] FlexRay. FlexRay Communications System Protocol Specification. Version 3.0.1., 2010.

[4] R. Makowitz and C. Temple, "FlexRay-a communication network for automotive control systems," in Proc. 2006 IEEE International Workshop on Factory Communication Systems, 2006, pp. 207-212.
[5] T. Pop, P. Pop, P. Eles et al., "Timing analysis of the FlexRay communication protocol," in Proc. 18th Euromicro Conference on Real-Time Systems, pp. 211-216.

[6] A. Hagiescu, U. D. Bordoloi, S. Chakraborty et al., "Performance analysis of flexray-based ECU networks," Design Automation Conference, 2007, pp. 284-289.

[7] P. Pop, P. Eles, and Z. Peng, "Bus access optimization for distributed embedded systems based on schedulability analysis," in Proc. Design, Automation and Test in Europe Conference and Exhibition, IEEE Computer Society, 2000, pp. 567-574.

[8] K. Schmidt and E. G. Schmidt, "Systematic message schedule construction for time-triggered CANS," IEEE Transactions on Vehicular Technology, vol. 56, no. 6, pp. 3431-3441, 2007.

[9] E. G. Schmidt and K. Schmidt, "Message scheduling for the flexray protocol: The dynamic segment," Vehicular Technology IEEE Transactions on, vol. 58, no. 5, pp. 2160-2169, 2009.

[10] B. J. Berwanger, M. Peteratzinger, and A. Schedl, FlexRay startet durch - FlexRay-Bordnetz für Fahrdynamik und Fahrerassistenz systeme. In Elektronik automotive: Sonderausgabe 7er BMW, 2008.

[11] P. Milbredt, B. Vermeulen, G. Tabanoglu et al., "Switched FlexRay: increasing the effective bandwidth and safety of FlexRay networks," in Proc. Design 2010 IEEE Conference on Emerging Technologies and Factory Automation.

[12] T. Schenkelaars, B. Vermeulen, and K. Goossens, "Optimal scheduling of switched FlexRay networks," Design Automation \& Test in Europe, 2011.

[13] M. Lukasiewycz, S. Chakraborty, and P. Milbredt, "FlexRay switch scheduling - A networking concept for electric vehicles," Design Automation \& Test in Europe, 2011.

[14] M. Grenier, L. Havet, and N. Navet, "Configuring the communication on FlexRay - The case of the static segment," European Congress Embedded Real Time Software, 2008.

[15] S. Ding, X. Yin, H. Xu et al., "A hybrid GA-based scheduling method for static segment in Flexray systems," in Proc. 2010 Chinese Control and Decision Conference, pp. 1548-1552, 2010.

[16] K. Schmidt and E. G. Schmidt, "Message scheduling for the flexray protocol: The static segment," IEEE Transactions on Vehicular Technology, vol. 58, no. 5, pp. 2170-2179, 2009.

[17] M. Lukasiewycz, M. Gla et al., "FlexRay schedule optimization of the static segment," in Proc. the 7th IEEE/ACM International Conference on Hardware/Software Codesign and System Synthesis, pp. 363-372, 2009.

[18] I. Park and M. Sunwoo, FlexRay Network Parameter Optimization Method for Automotive Application, IEEE Transactions, vol. 58, no. 4 pp. 1449-1459, 2011. 


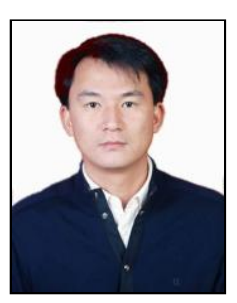

Yi-Nan Xu was born at Jilin province of China Currently, he is an associate professor of the division of electronic and communication engineering of Yanbian University, Yanji, China.

$\mathrm{He}$ received the $\mathrm{Ph} . \mathrm{D}$. degree in electrical engineering from the Chonbuk National University, Korea, in 2009. Corresponding author.

His research interests include the in-vehicle networks and automobile electronic control.

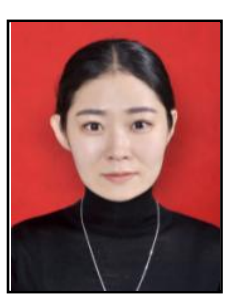

Xiang-Qi Kong was born at Jilin province of China. She completed bachelor degree in the field of electronics and telecommunication, Yanbian University, China, in 2016.

She is still pursuing her Master Degree in the school of Yanbian University, Yanji, China.

Her research interests include the In-vehicle FlexRay network.

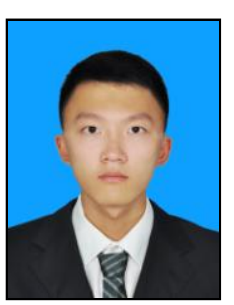

Meng-Zhuo Liu was born at Jilin province of China. He studied his undergraduate course at division of electronics engineering, Yanbian University, Yanji, China. He will still pursuing her Master Degree in the school of Yanbian University, Yanji, China

His research interests the In-vehicle FlexRay network.

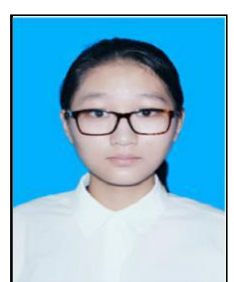

Shu-Wen Xue was born at Fujian province of China She studied her undergraduate course at division of electronics engineering, Yanbian University, Yanji, China. At the same time, she is a student of innovation.

Her research interests include the In-vehicle FlexRay network.

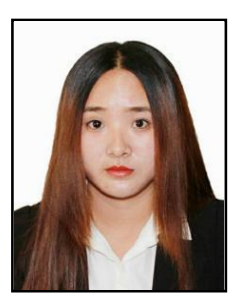

Zhe-Yu Sun was born at Inner Mongolia Autonomous region of China. She studied her undergraduate course at division of electronics engineering, Yanbian University, Yanji, China. At the same time, she is a student of innovation.

Her research interests include the automobile electronic control. 